\title{
Intraperitoneal chemotherapy in the management of ovarian cancer: focus on carboplatin
}

\author{
Maurie Markman \\ University of Texas MD Anderson \\ Cancer Center, Houston, Texas, USA
}

\begin{abstract}
Both pre-clinical studies and phase 1-2 clinical trials have provided strong support for the potential role of regional drug delivery in the management of epithelial ovarian cancer, a disease process whose major manifestations remain largely localized to the peritoneal cavity in the majority of individuals with this malignancy. The results of 3 phase 3 randomized trials have revealed the favorable impact of primary cisplatin-based intraperitoneal chemotherapy in women who initiate drug treatment with small-volume residual ovarian cancer following an attempt at optimal surgical cytoreduction. Concerns have been raised regarding the toxicity of regional treatment, particularly the side-effect profile associated with cisplatin. One rational approach to improving the tolerability of intraperitoneal chemotherapy is to substitute carboplatin for cisplatin. This review discusses the rationale for and data supporting regional treatment of epithelial ovarian cancer, and highlights the potential role for intraperitoneal carboplatin in this clinical setting.
\end{abstract}

Keywords: ovarian cancer, intraperitoneal chemotherapy, cisplatin, carboplatin

Due to its location in the peritoneal cavity, ${ }^{1,2}$ many have considered direct intraperitoneal delivery of anti-neoplastic agents a rational approach to the management of ovarian cancer. In fact, reports in the early days of the development of cytotoxic chemotherapeutic agents revealed the potential for this strategy to improve symptoms in this malignancy, particularly those associated with the accumulation of malignant ascites. ${ }^{3,4}$

Unfortunately, those initial experiences essentially failed to demonstrate superior efficacy associated with regional drug administration, compared to systemic (intravenous or oral) delivery, and the additional time and effort required made this a rather unattractive management option. Further, for certain drugs (eg, cytotoxic alkylating agents with vesicant properties), substantial local toxicity (eg, abdominal pain, adhesion formation leading to bowel obstruction) could be added to the known existing side effects of a particular drug (eg, emesis, bone marrow suppression). ${ }^{5,6}$

\section{"Dedrick model" of regional treatment of ovarian cancer}

In the late 1970s, Robert Dedrick and his colleagues at the National Cancer Institute (Bethesda, MD, USA), published a landmark theoretical modeling study which predicted that for anti-neoplastic agents possessing particular biological properties there would be a major pharmacokinetic advantage for exposure of the drug to cancer present within the peritoneal cavity following regional delivery, compared to systemic administration of the same agent. ${ }^{7}$ These provocative data and subsequently conducted pre-clinical evaluations provided a strong impetus for clinical investigators to renew their interest in exploring the concept of intraperitoneal treatment in the management of ovarian cancer. 


\section{Rational selection of anti-neoplastic agents for intraperitoneal chemotherapy}

Based on both the preclinical experience and the results of early phase clinical trials a number of features can be identified that would predict a particular anti-neoplastic drug might exhibit favorable properties if utilized via the intraperitoneal route.

First, it is critical that the agent is not a vesicant or major irritant, as it has been clearly noted the resulting inflammation can cause considerable local pain and subsequent adhesion formation. ${ }^{5}$ This event may interfere with drug distribution throughout the cavity, negatively influencing the efficacy of regional treatment. Further, in addition to the acute reversible effects, more serious toxicity can develop (eg, bowel obstruction leading to required surgical intervention).

Second, agents that are slowly removed from the peritoneal cavity and rapidly cleared from the systemic circulation will exhibit the greatest pharmacokinetic advantage following regional delivery. In this regard, drugs that are rapidly and extensively metabolized into non-toxic metabolites during their first-pass through the liver are particularly attractive for intraperitoneal administration, as it has long been recognized that drug uptake from the cavity is largely via the portal circulation., ${ }^{8,9}$

Third, preclinical data demonstrating that an agent is biologically active against the particular malignancy (eg, ovarian cancer), and that the activity can be substantially enhanced when the malignant cells are exposed to higher peak concentrations of drug, or for considerably longer periods of time (greater area-under-the concentration-versus-time curve [AUC]), than can safely be achieved following systemic delivery, provides a strong rationale for examining regional administration in the clinical setting. ${ }^{10}$

\section{Phase I trials of intraperitoneal therapy in ovarian cancer}

Over the last several decades, a rather large number of anti-neoplastic drugs have been examined for their safety and pharmacokinetic properties when delivered by the intraperitoneal route (Table 1). ${ }^{11}$ Importantly, the relative limited local toxicity associated with certain agents (including cisplatin and carboplatin) was confirmed in these studies. ${ }^{11,12-18}$ although this critically relevant finding was not universal (eg, pain associated with intraperitoneal doxorubicin). ${ }^{19}$

As predicted by the "Dedrick Model", those drugs known to undergo extensive metabolism during their first pass
Table I Pharmacokinetic advantage associated with intraperitoneal delivery of anti-neoplastic agents with known activity in ovarian cancer

\begin{tabular}{ll}
\hline Ratio: peak levels peritoneal cavity to systemic circulation \\
\hline Cisplatin & 20 \\
Carboplatin & 18 \\
Paclitaxel & 1000 \\
Doxorubicin & 470 \\
Melphalan & 80 \\
5-fluorouracil & 300 \\
\hline
\end{tabular}

through the liver have been shown to possess the greatest pharmacokinetic advantage following intraperitoneal administration. ${ }^{11}$ However, data for agents with more modest differences between total or peak exposures of the peritoneal and systemic compartments to the biologically active anti-neoplastic agents (eg, 10- to 20-fold for cisplatin, carboplatin) are of considerable interest and potential clinical relevance because these concentrations are simply not realistically attainable within the systemic compartment due to production of severe side effects (eg, bone marrow suppression; renal toxicity and neurotoxicity).

Further, provocative pre-clinical data for the platinum agents had revealed that resistance to these drugs can actually be quite "relative", and may conceivably be overcome by exposing tumor cells to only modestly higher (eg, 2- to 4-fold) concentrations of the drugs compared to that documented to be within the systemic compartment following standard systemic treatment. ${ }^{20}$ Unfortunately, extensive prior clinical experience has revealed that even "doubling the dose" and concentration of cisplatin (eg, $75 \mathrm{mg} / \mathrm{m}^{2}$ to $150 \mathrm{mg} / \mathrm{m}^{2}$ ) or carboplatin (AUC 6 to AUC 12), and administering multiple cycles of such a regimen, would unquestionably lead to unacceptable toxicity. However, these higher concentrations, as well as the increased peak levels and AUCs are theoretically rather easily achievable within the confines of the peritoneal cavity following regional administration of the platinum drugs.

\section{Depth of drug penetration: the major limiting factor associated with regional chemotherapy}

The early phase 1 clinical trials provided a strong rationale for further exploration of regional treatment of ovarian cancer. However, extensive pre-clinical experience suggested that despite the impressive pharmacokinetic advantage associated with regional therapy this management approach would find 
its greatest utility in the setting of small volume residual macroscopic cancer or microscopic disease only, due to the very limited ability of such drugs to penetrate into solid tissue. $^{21-24}$

Such penetration was observed in several modeling systems to range from a few cell layers to 1 to $2 \mathrm{~mm}$ from the cell surface. Of note, in a series of studies specifically examining the penetration of cisplatin in a rat model, the relative advantage of exposure to platinum, associated with intraperitoneal compared to systemic delivery, was a maximum of approximately a millimeter from the cell surface. ${ }^{22}$

These data would indicate that patients with large volume residual ovarian cancer would be unlikely to benefit from intraperitoneal delivery because the high concentrations of drugs measured within the cavity itself would not reach very deep into any residual tumor. However, one might also speculate that if a malignant mass lesion was substantially reduced in volume by exposure to biologically active systemic therapy regional drug delivery may be effective in subsequent courses against the smaller residual disease. ${ }^{25}$

Further, since it is known that platinum (both cisplatin and carboplatin) reaches the systemic compartment in essentially full concentrations following regional administration (largely due to the fact there is very limited metabolism of the agents within the liver), it is not unreasonable to consider the administration of a platinum drug intraperitoneally even in the setting of larger volume cancer. ${ }^{12-18}$ This conclusion follows from the hypothesis that there will be satisfactory concentrations of the drug reaching tumor by capillary flow, and with subsequent courses the smaller volume residual tumor may benefit from substantially greater local exposure associated with the intraperitoneal drug instillation. Of course, this is a hypothesis that is testable in a randomized phase 3 clinical trial.

\section{Phase 2 trial experience with intraperitoneal chemotherapy of ovarian cancer}

Not surprisingly, due it its established role in the management of ovarian cancer, the majority of experience examining regional delivery of anti-neoplastic drug therapy in ovarian cancer has focused on the platinum agents, principally cisplatin. ${ }^{11,26}$ Further, most of the trials have been in the second-line setting, following primary platinum-based systemic chemotherapy.

These circumstances permit an interesting perspective, in that is it possible to inquire about the level of biological activity observed with regional platinum delivery, compared to that of an individual patient's prior experience with intravenous platinum. One published study specifically examined this question and noted that tumors that had not previously exhibited tumor regression to the concentrations of platinum attainable with systemic therapy rarely responded to regional treatment (with activity documented at surgical reassessment). ${ }^{26}$ This was the case even if the patient had only very small volume residual cancer when the second-line cisplatin-based intraperitoneal treatment program was initiated.

Conversely, in patients whose cancers had achieved partial responses to prior platinum therapy, as many as $30 \%$ to $40 \%$ of such individuals were found to have attained a surgically documented complete response to intraperitoneal cisplatin. Of interest, these provocative data support the general concept that the high concentrations of platinum noted following regional treatment are able to overcome a modest level of existing resistance. However, unfortunately, the attainable concentrations are unable to influence major inherent platinum resistance.

Intraperitoneal carboplatin was also examined in several phase 2 trials in women with ovarian cancer in the secondline setting. ${ }^{14,27-30}$ In fact, overall, the drug demonstrated measurable biological activity quite comparable to that seen with cisplatin. This observation is not surprising in view of the known equivalence of the two platinum agents in advanced ovarian cancer when delivered systemically at optimal doses.

One retrospective analysis examining a single institutional experience employing either intraperitoneal cisplatin or carboplatin-based chemotherapy in the second-line setting noted similar surgically defined objective response rates to the two strategies in individuals who initiated chemotherapy with microscopic residual disease. ${ }^{31}$ However, a lower response rate was observed with carboplatin in women with any residual macroscopic cancer when the regional treatment program was initiated.

This clinical experience was similar to one reported pre-clinical evaluation that suggested the amount of measurable platinum within macroscopic tumors was lower with carboplatin than cisplatin following regional administration, ${ }^{32}$ but it is important to note these data have not been confirmed in any other pre-clinical model, or clinical experience with the two platinum strategies. Further, there have been no phase 3 randomized trials in the second-line setting to confirm (or refute) the utility of regional drug delivery with either cisplatin or carboplatin (or any other agent) in this patient population. 
Intraperitoneal carboplatin has also been examined in the phase 2 setting as a component of primary chemotherapy of advanced ovarian cancer. ${ }^{14,33,34}$ These non-randomized studies demonstrate the feasibility of the approach, and interesting survival data have been reported. What is missing are phase 3 trial data employing the agent in this manner.

\section{Phase 3 trials of cisplatin-based primary chemotherapy of small volume residual advanced ovarian cancer}

Several phase 3 randomized trials have now been reported that explored the clinical utility of regional cisplatin chemotherapy employed as primary chemotherapy of small volume residual advanced ovarian cancer following an attempt at maximal cytoreductive surgery. ${ }^{35-37}$ Three of these studies were of sufficient sample size and design to potentially provide definitive answers to the question of the utility of regional cisplatin in this setting (Table 2).

The first trial, conducted by the Southwest Oncology Group (SWOG) and the Gynecologic Oncology Group (GOG) compared the same dose $\left(100 \mathrm{mg} / \mathrm{m}^{2}\right)$ of either intraperitoneal or intravenous cisplatin. ${ }^{35}$ All patients entered into this study also received intravenous cyclophosphamide. The largest residual tumor mass size permitted entry into this trial was $20 \mathrm{~mm}$. Patients randomized to the intraperitoneal study arm experienced more abdominal discomfort (generally mild to moderate in severity), but no increase in severe clinical toxicity or treatment-related deaths. Further, there was a reduced incidence of neutropenia and tinnitus associated with local drug delivery. Of greatest importance, the study revealed a statistically significant improvement in overall survival associated with regional delivery of cisplatin in this clinical setting (Table 2).

A second primary ovarian cancer regional chemotherapy trial was then conducted by the GOG and SWOG, with the addition of the Eastern Cooperative Oncology Group (ECOG) which again compared intraperitoneal cisplatin to intravenous cisplatin, but in this study all patients also received intravenous paclitaxel, rather than intravenous cyclophosphamide. ${ }^{36}$ Of note, the largest size of a residual mass lesion permitted entry into this trial was $10 \mathrm{~mm}$.

This study added another novel component, based on the previously stated belief that regional drug delivery would be most relevant in those patients with the smallest residual tumor volumes. Thus, prior to the administration of regional chemotherapy, patients randomized to this study arm received 2 cycles of "moderately high dose" intravenous carboplatin (AUC 9) designed to "chemically debulk" any residual macroscopic cancer remaining after the initial cytoreductive surgery. ${ }^{38}$

Unfortunately, the two courses of intravenous carboplatin produced an unexpectedly high incidence of severe thrombocytopenia, such that $19 \%$ of patients received two or fewer courses of intraperitoneal drug delivery. This outcome was a direct result of carboplatin-associated bone marrow suppression and not local toxic effects of the regional treatment program. Despite this fact, this study revealed improved progression-free survival and a borderline improvement in overall survival in the experimental regional treatment program, compared to the all-intravenous therapeutic regimen (Table 2).

The most recently reported randomized ovarian cancer regional chemotherapy study again compared intravenous to intraperitoneal cisplatin, but patients in the intraperitoneal chemotherapy arm were also administered intraperitoneal paclitaxel. ${ }^{37}$ All patients in the trial received intravenous paclitaxel. As with the previously discussed study, the largest size of any residual mass permitted entry into this trial was $10 \mathrm{~mm}$. This study revealed a statistically significant 16-month median improvement in overall survival associated with the regional therapy program, in addition to a documented improvement in progression-free survival.

Table 2 Phase 3 randomized trials comparing intraperitoneal (ip) versus intravenous (iv) cisplatin-based drug delivery employed as primary chemotherapy of small-volume residual advanced epithelial ovarian cancer

\begin{tabular}{|c|c|c|}
\hline & Progression-free survival (median) & Overall survival (median) \\
\hline $\begin{array}{l}\text { ip versus iv cisplatin (all patients also received } \\
\text { iv cyclophosphamide) }\end{array}$ & - & 48 vs $4 I$ months $(p=0.02 ; \mathrm{HR} 0.76)$ \\
\hline $\begin{array}{l}\text { ip versus iv cisplatin }{ }^{\text {a }} \text { all patients also received } \\
\text { iv paclitaxel) }{ }^{36}\end{array}$ & 28 vs 22 months $(p=0.01 ;$ HR 0.78$)$ & 63 vs 52 months $(p=0.05 ;$ HR $0.8 \mathrm{I})$ \\
\hline $\begin{array}{l}\text { ip versus iv cisplatin }{ }^{\mathrm{b}} \text { (all patients also received } \\
\text { iv paclitaxel) } \text { (37 }^{37}\end{array}$ & 23.8 vs 18.3 months $(P=0.05 ;$ HR 0.77$)$ & 65.6 vs 49.7 months $(p=0.03 ;$ HR 0.73$)$ \\
\hline
\end{tabular}

a Patients in the ip treatment arm also received two cycles of moderately high dose iv carboplatin prior to the delivery of regional chemotherapy.

bPatients in the ip treatment arm received ip paclitaxel in addition to ip cisplatin. 
The intraperitoneal regimen was found to cause more toxicity and less than one half of the treated patient population was able to complete the entire planned 6 treatment cycles. Of interest, this study included a prospective evaluation of measures of quality of life. This analysis revealed inferior quality of life associated with the regional program during therapy, but importantly at 1-year follow-up there was essentially no difference between the two study arms.

One highly provocative question resulting from this third randomized trial is the issue of whether the documented improvement in overall survival observed following intraperitoneal drug delivery might have been even greater if a higher percentage of women had been able to receive the entire planned 6 treatment courses. Alternatively, it could be argued that the majority of the benefits achieved from regional platinum delivery may be observed if women are administered only 3 or 4 such cycles. Again, these questions remain interesting hypotheses that may be tested in future phase 3 randomized trials.

\section{Rational use of intraperitoneal chemotherapy in epithelial ovarian cancer}

Based on existing data, where is it appropriate to consider the use of intraperitoneal chemotherapy in the routine management of ovarian cancer?

Existing evidence-based (randomized phase 3 trial) data provide strong support for the conclusion that patients with small volume residual advanced ovarian cancer (largest tumor mass $<10 \mathrm{~mm}$ in maximum diameter) should be offered the option to receive a cisplatin-based intraperitoneal strategy, assuming there are no medical contraindications to receiving cisplatin (eg, renal insufficiency; pre-existing neuropathy) or to receiving regional treatment (eg, presence of extensive adhesions at the time of prior surgery). ${ }^{35-37,39,40}$ Further, while a patient can certainly elect to not receive this treatment, based on the time, effort, and potential additional morbidity associated with the approach, it can also be argued that oncologists whose particular practice setting prevents their being able to offer such therapy should consider referral (where feasible) of an ovarian cancer patient who may benefit from intraperitoneal drug delivery to an oncology practice where this strategy can be administered.

Intraperitoneal chemotherapy may also be rationally offered to women with advanced ovarian cancer who have been managed primarily with a neoadjuvant approach, but where an initial excellent response to chemotherapy has led to surgery and no or minimal residual tumor volume following the completion of this interval cytoreductive procedure. ${ }^{41}$ Here one can argue that the high local concentrations achievable with intraperitoneal treatment may potentiate the effects of the chemotherapeutic agents already documented to be highly biologically active in the particular individual's cancer. The ultimate benefits of this approach compared to the continuation of systemic chemotherapy is worthy of investigation in a phase 3 randomized trial. Of note, such a trial is currently planned to be conducted by the Gynecologic Cancer Intergroup, an international consortium of gynecologic cancer clinical investigators.

Intraperitoneal anti-neoplastic drug delivery may also be considered in the setting of an ovarian cancer patient with a high grade malignancy who has achieved a surgically defined complete response to primary systemic chemotherapy, where it is known the ultimate risk of recurrence is very high. While interesting phase 2 trial data supporting this approach have been presented, ${ }^{42}$ the few very underpowered phase 3 trials that were designed to evaluate this approach have not provided evidence for the utility of this form of consolidation therapy. ${ }^{43}$

Based on the results of the previously noted non-randomized phase 2 trials examining platinum-based second-line therapy in ovarian cancer, it is not unreasonable to consider this strategy in a woman who has achieved a major partial response following primary systemic platinum chemotherapy. ${ }^{11,44-46}$ However, again, no randomized phase 3 trial data are available to demonstrate the superiority of this approach compared to continuation of systemic therapy.

Finally, as it is known that patients with "high risk" early stage ovarian cancer have a $30 \%$ to $50 \%$ chance of experiencing recurrence of the disease process, and those recurrences are largely within the peritoneal cavity, it is perhaps reasonable to consider delivering some, or perhaps all, of a planned adjuvant chemotherapy approach via the intraperitoneal route. As previously stated, the administration of either intraperitoneal cisplatin or carboplatin will lead to adequate systemic drug concentrations, and also substantially higher platinum concentrations within the peritoneal cavity.

\section{A role for intraperitoneal carboplatin}

As outlined within this text, the large majority of currently reported trials examining regional drug delivery in ovarian cancer have employed cisplatin, rather then carboplatin. This is largely due to the fact the early studies of intraperitoneal chemotherapy were initiated before the widespread use of carboplatin, and subsequent evidence of the equivalence 
of the two platinum drugs when delivered systemically in advanced ovarian cancer.

Is it reasonable to simply substitute carboplatin in any regimen where cisplatin has been utilized?

Extensive experience with systemically administered carboplatin in ovarian cancer has revealed the two agents have essentially equivalent biological activity, but with quite different toxicity profiles. ${ }^{47-49}$ The modestly reduced potential for the development of neuropathy associated with carboplatin, compared to cisplatin, is particularly relevant when the platinum agent is combined with another neurotoxic drug, such as a taxane. A "carboplatin plus paclitaxel" chemotherapy regimen is easily administered in the out-patient setting, and this program continues to be an overwhelmingly favorite primary strategy among oncologists treating women systemically for ovarian cancer.

As previously noted, phase 2 trials of intraperitoneal carboplatin in ovarian cancer have reported favorable survival and toxicity outcomes, but there remains a notable absence of randomized phase 3 trial data employing the newer platinum drug delivered by this route. The GOG plans to examine intraperitoneal carboplatin in a phase 3 trial in the future, but it is unknown at this point in time when data from this experience will be available.

For the present, it is reasonable to consider several options when contemplating the use of intraperitoneal carboplatin in ovarian cancer: $:^{50,51}$

1. Substitution of carboplatin for cisplatin in all patients receiving intraperitoneal chemotherapy:

While certainly not an irrational option, if an oncologist elects to simply substitute carboplatin for cisplatin, for example in a patient with small volume residual advanced ovarian cancer after primary surgical cytoreduction, it is incumbent upon the physician to inform the patient that the evidence-based studies that documented the utility of regional therapy actually employed cisplatin. Further, the specific justification for utilizing carboplatin, rather than cisplatin, should be explained to the patient.

2. Substitution of carboplatin if excessive toxicity with cisplatin is observed:

In this situation, the physician will attempt to initially deliver intraperitoneal cisplatin but if the patient experiences excessive side effects (eg, emesis), the next course can be administered with intraperitoneal carboplatin. One can rationally argue that if the choice is between switching therapy to either intravenous carboplatin or intraperitoneal carboplatin, there is no reason to believe regional carboplatin delivery will be less effective, and it may be more effective than systemic administration of the agent.

3. Plan to deliver a limited number of courses of both intraperitoneal cisplatin and carboplatin:

This strategy recognizes the fact it is difficult for the majority of patients to tolerate 6 cycles of a cisplatinbased intraperitoneal chemotherapy program, despite the fact the data demonstrating a survival benefit were based on delivery of this agent. Thus, it might be planned that a woman with small volume residual advanced ovarian cancer will be treated with 2 to 3 cycles of intraperitoneal cisplatin (depending on tolerability), and then switched to intraperitoneal carboplatin (3-4 cycles) to complete the treatment program.

The optimal dose of intraperitoneal carboplatin also remains to be defined, but on the basis of existing data (both systemic and regional) it is rational to propose delivering the agent by this route at an AUC (area-under-the-concentrationversus-time curve) of 5 or $6 .^{14,47-51}$

\section{Conclusion}

The intraperitoneal delivery of cisplatin has been demonstrated in several evidence-based randomized phase 3 trials to improve overall survival when employed as first-line chemotherapy treatment of women with small volume residual advanced ovarian cancer. Despite this fact, many oncologists remain reluctant to routinely employ this approach, often because of the well-recognized systemic toxicity associated with cisplatin.

The use of intraperitoneal carboplatin has a particular appeal in this setting, as the drug is well established as the primary platinum agent employed in the management of women with ovarian cancer, and it has been documented to have a more favorable toxicity profile compared to the older platinum. Another very pragmatic attraction of intraperitoneal carboplatin is that use of the agent makes it easier to deliver regional therapy in the setting of a busy oncology practice (eg, less actual required treatment time; reduced risk of troublesome toxicities). Unfortunately, there remain no phase 3 trial data documenting the equivalence of intraperitoneal cisplatin and carboplatin, or the superiority of intraperitoneal carboplatin compared to intravenous carboplatin.

Planned trials will hopefully provide definitive answers to this important question. For the present, it is not unreasonable to employ intraperitoneal carboplatin in the management of ovarian cancer after considering the limitations of existing data, and the potential toxicity of cisplatin-based therapy that may be, or has been, encountered in an individual patient. 


\section{Disclosures}

The author has no conflicts of interest to disclose.

\section{References}

1. Bergman F. Carcinoma of the ovary. A clinicopathological study of 86 autopsied cases with special reference to mode of spread. Acta Obstet Gynecol Scand. 1966;45:211-231.

2. Dauplat J, Hacker NF, Nieberg RK, Berek JS, Rose TP, Sagae S. Distant metastases in epithelial ovarian carcinoma. Cancer. 1987;60:1561-1566.

3. Weisberger AS, Levine B, Storaasli JP. Use of nitrogen mustard in treatment of serous effusions of neoplastic origin. JAMA. 1955;159:1704-1707.

4. Suhrland LG, Weisberger AS. Intracavitary 5-fluorouracil in malignant effusions. Arch Intern Med. 1965;116:431-433.

5. Litterst CL, Collins JM, Lowe MC, Arnold ST, Powell DM, Guarino AM. Local and systemic toxicity resulting from large-volume Ip administration of doxorubicin in the rat. Cancer Treat Rep. 1982;66:157-161.

6. Markman M, George M, Hakes T, et al. Phase II trial of intraperitoneal mitoxantrone in the management of refractory ovarian cancer. J Clin Oncol. 1990;8:146-150.

7. Dedrick RL, Myers CE, Bungay PM, DeVita VT Jr. Pharmacokinetic rationale for peritoneal drug administration in the treatment of ovarian cancer. Cancer Treat Rep. 1978;62:1-9.

8. Kraft AR, Tompkins RK, Kaplan RA. Peritoneal electrolyte absorption: Analysis of portal, systemic venous and lymphatic transport. Surgery. 1968;64:148-153.

9. Lukas G, Brindle SD, Greengard P. The route of absorption of intraperitoneally administered compounds. J Pharmacol ExpTher. 1971;178:562-564.

10. Alberts DS, Young L, Mason N, Salmon SE. In vitro evaluation of anticancer drugs against ovarian cancer at concentrations achievable by intraperitoneal administration. Semin Oncol. 1985;12:38-42.

11. Markman M. Intraperitoneal antineoplastic drug delivery: rationale and results. Lancet Oncol. 2003;4:277-283.

12. Casper ES, Kelsen DP, Alcock NW, Lewis JL, Jr. Ip cisplatin in patients with malignant ascites: pharmacokinetic evaluation and comparison with the IV route. Cancer Treat Rep. 1983;67:235-238.

13. Degregorio MW, Lum BL, Holleran WM, Wilbur BJ, Sikic BI. Preliminary observations of intraperitoneal carboplatin pharmacokinetics during a phase I study of the Northern California Oncology Group. Cancer Chemother Pharmacol. 1986;18:235-238.

14. Fujiwara K, Markman M, Morgan M, Coleman RL. Intraperitoneal carboplatin-based chemotherapy for epithelial ovarian cancer. Gynecol Oncol. 2005;97:10-15.

15. Howell SB, Pfeifle CL, Wung WE, et al. Intraperitoneal cisplatin with systemic thiosulfate protection. Ann Intern Med. 1982;97:845-851.

16. Elferink F, van der Vijgh WJ, Klein I, Bokkel Huinink WW, Dubbelman R, McVie JG. Pharmacokinetics of carboplatin after intraperitoneal administration. Cancer Chemother Pharmacol. 1988;21:57-60.

17. Miyagi Y, Fujiwara K, Kigawa J, et al. Intraperitoneal carboplatin infusion may be a pharmacologically more reasonable route than intravenous administration as a systemic chemotherapy. A comparative pharmacokinetic analysis of platinum using a new mathematical model after intraperitoneal vs intravenous infusion of carboplatin-a Sankai Gynecology Study Group (SGSG) study. Gynecol Oncol. 2005;99:591-596.

18. Pretorius RG, Hacker NF, Berek JS, et al. Pharmacokinetics of Ip cisplatin in refractory ovarian carcinoma. Cancer Treat Rep. 1983;67:1085-1092.

19. Ozols RF, Young RC, Speyer JL, et al. Phase I and pharmacological studies of adriamycin administered intraperitoneally to patients with ovarian cancer. Cancer Res. 1982;42:4265-4269.

20. Andrews PA, Velury S, Mann SC, Howell SB. cis-Diamminedichlor oplatinum(II) accumulation in sensitive and resistant human ovarian carcinoma cells. Cancer Res.1988;48:68-73.
21. Durant RE. Flow cytometry studies of intracellular adriamycin in multicell spheroids in vitro. Cancer Res.1981;41:3495-3498.

22. Los G, Mutsaers PHA, van der Vijgh WJF, Baldew GS, de Graaf PW, McVie JG. Direct diffusion of cis-diamminedichloroplatinum(II) in intraperitoneal rat tumors after intraperitoneal chemotherapy: A comparison with systemic chemotherapy. Cancer Res.1989;49:3380-3384.

23. Nederman T, Carlsson J. Penetration and binding of vinblastine and 5-fluorouracil in cellular spheroids. Cancer Chemother Pharmacol. 1984;13:131-135.

24. West GW, Weichselbaum R, Little JB. Limited penetration of methotrexate into human osteosarcoma spheroids as a proposed model for solid tumor resistance to adjuvant chemotherapy. Cancer Res. 1980;40:3665-3668.

25. Markman M. Critical thinking: an essential role in both the conduct and interpretation of gynecologic cancer clinical research. Gynecol Oncol. 2008;108:462-465.

26. Markman M, Reichman B, Hakes T, et al. Responses to secondline cisplatin-based intraperitoneal therapy in ovarian cancer: influence of a prior response to intravenous cisplatin. J Clin Oncol. 1991;9:1801-1805.

27. Bruzzone M, Rubagotti A, Gadducci A, et al. Intraperitoneal carboplatin with or without interferon-alpha in advanced ovarian cancer patients with minimal residual disease at second look: a prospective randomized trial of 111 patients. G.O.N.O. Gruppo Oncologic Nord Ovest. Gynecol Oncol. 1997;65:499-505.

28. McClay EF, Braly PD, Kirmani S, et al. A phase II trial of intraperitoneal high-dose carboplatin and etoposide with granulocyte macrophagecolony stimulating factor support in patients with ovarian carcinoma. Am J Clin Oncol. 1995;18:23-26.

29. Markman M, Reichman B, Hakes T, et al. Phase 2 trial of intraperitoneal carboplatin and etoposide as salvage treatment of advanced epithelial ovarian cancer. Gynecol Oncol. 1992;47:353-357.

30. Speyer JL, Beller U, Colombo N, et al. Intraperitoneal carboplatin: favorable results in women with minimal residual ovarian cancer after cisplatin therapy. J Clin Oncol. 1990;8:1335-1341.

31. Markman M, Reichman B, Hakes T, et al. Evidence supporting the superiority of intraperitoneal cisplatin compared to intraperitoneal carboplatin for salvage therapy of small-volume residual ovarian cancer. Gynecol Oncol. 1993;50:100-104.

32. Los G, Verdegaal EM, Mutsaers PH, McVie JG. Penetration of carboplatin and cisplatin into rat peritoneal tumor nodules after intraperitoneal chemotherapy. Cancer Chemother Pharmacol. 1991;28:159-165.

33. Fujiwara K, Sakuragi N, Suzuki S, et al. First-line intraperitoneal carboplatin-based chemotherapy for 165 patients with epithelial ovarian carcinoma: results of long-term follow-up. Gynecol Oncol. 2003;90:637-643.

34. Nagao S, Fujiwara K, Ohishi R, et al. Combination chemotherapy of intraperitoneal carboplatin and intravenous paclitaxel in suboptimally debulked epithelial ovarian cancer. Int J Gynecol Cancer. 2008;18:1210-1214

35. Alberts DS, Liu PY, Hannigan EV, et al. Intraperitoneal cisplatin plus intravenous cyclophosphamide versus intravenous cisplatin plus intravenous cyclophosphamide for stage III ovarian cancer. $N$ Engl $J$ Med. 1996;335:1950-1955.

36. Markman M, Bundy BN, Alberts DS, et al. Phase III trial of standard-dose intravenous cisplatin plus paclitaxel versus moderately high-dose carboplatin followed by intravenous paclitaxel and intraperitoneal cisplatin in small-volume stage III ovarian carcinoma: an intergroup study of the Gynecologic Oncology Group, Southwestern Oncology Group, and Eastern Cooperative Oncology Group. J Clin Oncol. 2001;19:1001-1007.

37. Armstrong DK, Bundy B, Wenzel L, et al. Intraperitoneal cisplatin and paclitaxel in ovarian cancer. $N$ Engl J Med. 2006;354:34-43.

38. Shapiro F, Schneider J, Markman M, et al. High-intensity intravenous cyclophosphamide and cisplatin, interim surgical debulking, and intraperitoneal cisplatin in advanced ovarian carcinoma: a pilot trial with ten-year follow-up. Gynecol Oncol. 1997;67:39-45. 
39. Elit L, Oliver TK, Covens A, et al. Intraperitoneal chemotherapy in the first-line treatment of women with stage III epithelial ovarian cancer: a systematic review with metaanalyses. Cancer. 2007;109:692-702.

40. Hess LM, Ham-Hutchins M, Herzog TJ, et al. A meta-analysis of the efficacy of intraperitoneal cisplatin for the front-line treatment of ovarian cancer. Int J Gynecol Cancer. 2007;17:561-570.

41. Markman M, Belinson J. A rationale for neoadjuvant systemic treatment followed by surgical assessment and intraperitoneal chemotherapy in patients presenting with non-surgically resectable ovarian or primary peritoneal cancers. J Cancer Res Clin Oncol. 2005;131:26-30.

42. Barakat RR, Almadrones L, Venkatraman ES, et al. A phase II trial of intraperitoneal cisplatin and etoposide as consolidation therapy in patients with Stage II-IV epithelial ovarian cancer following negative surgical assessment. Gynecol Oncol. 1998;69:17-22.

43. Alberts DS, Hannigan EV, Liu PY, et al. Randomized trial of adjuvant intraperitoneal alpha-interferon in stage III ovarian cancer patients who have no evidence of disease after primary surgery and chemotherapy: An intergroup study. Gynecol Oncol. 2006;100:133-138.

44. Howell SB, Zimm S, Markman M, et al. Long-term survival of advanced refractory ovarian carcinoma patients with small-volume disease treated with intraperitoneal chemotherapy. J Clin Oncol. 1987;5:1607-1612.
45. Markman M, Reichman B, Hakes $T$, et al. Impact on survival of surgically defined favorable responses to salvage intraperitoneal chemotherapy in small-volume residual ovarian cancer. J Clin Oncol. 1992;10:1479-1484.

46. Recio FO, Piver MS, Hempling RE, Driscoll DL. Five-year survival after second-line cisplatin-based intraperitoneal chemotherapy for advanced ovarian cancer. Gynecol Oncol. 1998;68:267-273.

47. Covens A, Carey M, Bryson P, Verma S, Fung Kee FM, Johnston M. Systematic review of first-line chemotherapy for newly diagnosed postoperative patients with stage II, III, or IV epithelial ovarian cancer. Gynecol Oncol. 2002;85:71-80.

48. duBois A., Luck HJ, Meier W, et al. A randomized clinical trial of cisplatin/paclitaxel versus carboplatin/paclitaxel as first-line treatment of ovarian cancer. J Natl Cancer Inst. 2003;95:1320-1329.

49. Ozols RF, Bundy BN, Greer BE, et al. Phase III trial of carboplatin and paclitaxel compared with cisplatin and paclitaxel in patients with optimally resected stage III ovarian cancer: a Gynecologic Oncology Group study. J Clin Oncol. 2003;21:3194-3200.

50. Markman M, Walker JL. Intraperitoneal chemotherapy of ovarian cancer: a review, with a focus on practical aspects of treatment. J Clin Oncol. 2006;24:988-994.

51. Fujiwara K. Can carboplatin replace cisplatin for intraperitoneal use? Int J Gynecol Cancer. 2008;18 Suppl 1:29-32. 